Viebrock, Britta

\title{
Zur Professionalisierung von Lehrkräften im bilingualen Unterricht
}

Zeitschrift für interpretative Schul- und Unterrichtsforschung 3 (2014), S. 72-85

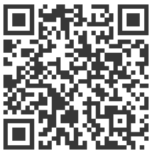

Quellenangabe/ Reference:

Viebrock, Britta: Zur Professionalisierung von Lehrkräften im bilingualen Unterricht - In: Zeitschrift für interpretative Schul- und Unterrichtsforschung 3 (2014), S. 72-85 - URN:

urn:nbn:de:0111-pedocs-160316 - DOI: 10.25656/01:16031

https://nbn-resolving.org/urn:nbn:de:0111-pedocs-160316

https://doi.org/10.25656/01:16031

in Kooperation mit / in cooperation with:

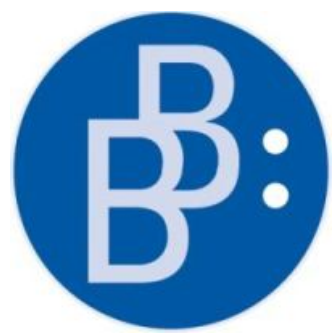

https://www.budrich.de

\section{Nutzungsbedingungen}

Gewährt wird ein nicht exklusives, nicht übertragbares, persönliches und beschränktes Recht auf Nutzung dieses Dokuments. Dieses Dokument ist ausschließlich für den persönlichen, nicht-kommerziellen Gebrauch bestimmt. Die Nutzung stellt keine Übertragung des Eigentumsrechts an diesem Dokument dar und gilt vorbehaltlich der folgenden Einschränkungen: Auf sämtlichen Kopien dieses Dokuments müssen alle Urheberrechtshinweise und sonstigen Hinweise auf gesetzlichen Schutz beibehalten werden. Sie dürfen dieses Dokument nicht in irgendeiner Weise abändern, noch dürfen Sie dieses Dokument für öffentliche oder kommerzielle Zwecke vervielfältigen, öffentlich ausstellen, aufführen, vertreiben oder anderweitig nutzen.

Mit der Verwendung dieses Dokuments erkennen Sie die Nutzungsbedingungen an.

\section{Terms of use}

We grant a non-exclusive, non-transferable, individual and limited right to using this document.

This document is solely intended for your personal, non-commercial use. Use of this document does not include any transfer of property rights and it is conditional to the following limitations: All of the copies of this documents must retain all copyright information and other information regarding legal protection. You are not allowed to alter this document in any way, to copy it for public or commercial purposes, to exhibit the document in public, to perform, distribute or otherwise use the document in public.

By using this particular document, you accept the above-stated conditions of use.

\section{Kontakt / Contact:}

\section{peDOcs}

DIPF | Leibniz-Institut für Bildungsforschung und Bildungsinformation Informationszentrum (IZ) Bildung

E-Mail:pedocs@dipf.de

Internet: www.pedocs.de

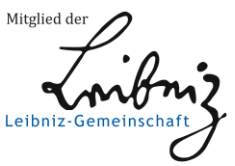




\section{Inhalt}

\section{ZISU}

\section{Zeitschrift für interpretative Schul- und Unterrichtsforschung}

\section{Editorial}

Andreas Bonnet, Uwe Hericks

\section{Thementeil}

Angelika Paseka, Jan-Hendrik Hinzke

Julia Košinár

Katharina Kunze

Martin Heinrich, Ann-Kathrin Arndt, Rolf Werning

Britta Viebrock

Andreas Bonnet, Uwe Hericks

Carolin Rotter

Afra Sturm, Nadja Lindauer
Professionalisierung und Deprofessionalisierung im Lehrer/innenberuf - Ansätze und Befunde aktueller empirischer Forschung

Der Umgang mit Dilemmasituationen -

Ein Beitrag zu Fragen der Professionalität von

Lehrpersonen und Lehramtsstudierenden

Die Bedeutung von Passungserfahrungen für

Professionalisierungsverläufe im Referendariat

Professionalisierungspotenziale und -probleme der sozialisatorischen Interaktion im Studienseminar

Von „Fördertanten“ und „Gymnasialempfehlungskindern“ - Professionelle Identitätsbehauptung von

Sonderpädagog/innen in der inklusiven Schule

Zur Professionalisierung von Lehrkräften im bilingualen Unterricht

„... kam grad am Anfang an die Grenzen“ Potenziale und Probleme von Kooperativem Lernen für die Professionalisierung von Englischlehrer/innen

Kompetent durch Migrationserfahrung? - Die Betonung des Migrationshintergrunds als Gefahr einer Deprofessionalisierung von Lehrkräften

Zwischen begrenztem Wissen und Widerspruch Zur Expertise von Kursleitenden im Bereich des 


\section{Diskussion}

Walter Herzog

Weshalb uns Hattie eine Geschichte erzählt - Oder:

130 Ein missglückter Versuch, den Erkenntnisstand der quantitativen Unterrichtsforschung zur Synthese zu bringen

\section{Rezensionen}

Rahel Hünig,

Was ist Unterricht?

Sascha Eberz

Rezension der gleichnamigen Tagung im

September 2013 in Halle

Oliver Hollstein

Zaborowski, Katrin Ulrike/Meier, Michael/

Breidenstein, Georg (2011): Leistungsbewertung

und Unterricht. Ethnographische Studien

zur Bewertungspraxis in Gymnasium und

Sekundarschule. Wiesbaden: VS Verlag für

Sozialwissenschaften.

Alexander Schuster,

Potenziale einer zentralen Verfügbarkeit von Daten

152

Doris Bambey

und Instrumenten im Forschungsdatenzentrum (FDZ)

Bildung 


\title{
Zur Professionalisierung von Lehrkräften im bilingualen Unterricht
}

\begin{abstract}
Zusammenfassung
Die Professionalisierung von Lehrkräften ist ein zentrales Thema in Erziehungswissenschaft und Fachdidaktik. Der vorliegende Beitrag ordnet die Ergebnisse eines Forschungsprojekts zu subjektiven didaktischen Theorien bilingualer Erdkundelehrkräfte in den Kontext der Professionalisierungsdebatte ein. Neben der Präsentation einzelner Forschungsergebnisse wird die Frage diskutiert, inwieweit das Konzept der subjektiven Theorien hilfreich ist für die Erfassung von Professionalisierungsprozessen. Dabei wird unterschieden zwischen einem „prescribed professionalism“, der sich aus verordneten bildungspolitischen Innovationen entwickelt, und einem „independent professionalism“, mit dem sich die Professionalisierungsprozesse einer bottom-up Entwicklung wie der des bilingualen Unterrichts aus der Schulpraxis heraus beschreiben lassen.

Schlagwörter: Didaktik des bilingualen (Erdkunde-)Unterrichts; bottom-up Innovationen; „independent professionalism“vs. „prescribed professionalism“; subjektive didaktische Theorien
\end{abstract}

\section{The professional development of CLIL teachers}

The professional development of teachers is an important issue in education and pedagogy as well as teaching theory and methodology. This paper discusses the results of a research project on teachers' subjective theories concerning content and language integrated learning (CLIL) in Geography in the light of the debate on professionalism. In addition to the presentation of individual research results and the teachers' prototypical argumentative structures it explores the question whether the concept 'subjective theories' is suitable for describing and analysing processes of increased professionalism. A distinction between ,prescribed professionalism' to describe outcomes of top-down innovations in education and ,independent professionalism' to describe outcomes of institutional bottom-up innovations such as CLIL is employed in order to show the scope of different processes of professionalism in the teaching profession.

Keywords: CLIL theory and methodology; bottom-up innovations in education; , independent professionalism'vs. , prescribed professionalism'; teachers' subjective theories

\section{Einleitung}

In der Diskussion um die Professionalisierung von Lehrkräften finden sich einerseits zahlreiche konzeptionelle Überlegungen, was Lehrerprofessionalität ausmacht. Andererseits wird dem Phänomen empirisch nachgegangen, vor allem in den Bereichen aufgesetzter bildungspolitischer Bestimmungen (Hinwendung zur Kompetenzorientierung, Bildungsstandards etc.), zu denen Lehrkräfte sich notgedrungen verhalten müssen. Auch in den Fachdidaktiken, für den vorliegenden Beitrag speziell in der Fremdsprachendidaktik und der Didaktik des bilingualen Unterrichts ${ }^{1}$, spielen Aspekte von Lehrerprofessio-

1 Im bilingualen Unterricht wird eine Fremdsprache als Arbeitssprache im Fachunterricht genutzt. Der international gebräuchliche Terminus ist Content and Language Integrated Learning (CLIL). Üblicherweise wird die Fremdsprache in ausgewählten Fächern genutzt (vgl. Breidbach/ Viebrock 2012). 
nalität eine Rolle. Allerdings werden sie selten explizit mit diesem Begriff in Verbindung gebracht (eine Ausnahme bilden die Arbeiten von Dirks 2000, 2004). Ein Grund hierfür mag sein, dass sich die Fremdsprachendidaktik bzw. die Überlegungen zum bilingualen Unterricht in erster Linie mit einem begrenzten, wenn auch zentralen Ausschnitt der Tätigkeit von Lehrer/innen befassen, nämlich der Inszenierung und Reflexion fachlicher und sprachlicher Lernprozesse, weniger aber mit den allgemeinpädagogischen und administrativen Aufgabenfeldern, welche der Lehrberuf darüber hinaus umfasst.

Vor diesem Hintergrund verfolgt der vorliegende Beitrag das Anliegen, die Ergebnisse eines Forschungsprojekts zu den subjektiven didaktischen Theorien bilingualer Erdkundelehrer/innen in den Kontext der Professionalisierungsdebatte einzuordnen und aufzuzeigen, inwieweit das Konzept der subjektiven Theorien hilfreich für die Erfassung von individuellen Professionalisierungsprozessen ist. Dazu wird zunächst ein Blick auf ausgewählte Professionalitätsmodelle sowie empirische Erkenntnisse zu den Auswirkungen pädagogischer Innovationen auf die Professionalisierung von Lehrer/ innen geworfen, bevor die Spezifika und Ergebnisse des Forschungsprojekts dargestellt werden. Eine Besonderheit des bilingualen Unterrichts ist es, dass es sich um eine Grassroots-Bewegung handelt, bei der Innovationen aus der Schulpraxis hervorgingen, bevor die Thematik in den wissenschaftlichen Diskurs Eingang gefunden hatte (vgl. Breidbach/Viebrock 2012). Ob und in welchem Umfang diese Tatsache Einfluss auf die Entwicklung professionellen Handelns nimmt, soll ebenfalls Gegenstand der folgenden Überlegungen sein.

\section{Professionalisierung im Lehrberuf}

Professionalität und Professionalisierung sind in den letzten zwei Jahrzehnten zu inflationären Begriffen mit zahlreichen Bedeutungsausprägungen geworden. Die Frage, inwieweit der Lehrberuf als Profession gelten kann und durch welche Merkmale sich professionelle Kompetenzen auszeichnen, wird kontrovers diskutiert. Grundsätzlich lassen sich vier Ansätze unterscheiden, die in unterschiedlicher Form für das hier beschriebene Forschungsprojekt relevant werden:

(1) In Anlehnung an das klassische Professionenkonzept ist der Lehrberuf als Profession ausgewiesen worden: „Kriterien für die Veredelung eines Berufs zur Profession sind eine Berufsausübung, die auf wissenschaftlichem Wissen gründet, eine freiberufliche Stellung, ,Autonomie bei der Formulierung von Standards der Berufsausübung und Ausbildung'; eine berufsständische Organisation, eine Berufsethik und die Klientenorientierung [...]. Eine eigene Fachsprache wird ebenfalls als Kriterium angeführt" (Rothland/Brüggemann 2011: 536f.). Zweifel an der professionellen Autonomie des Lehrberufs haben zur Weiterentwicklung des Konzepts geführt. Ebenso ist zu hinterfragen, für welche Bereiche das wissenschaftliche Wissen explizit ausgewiesen sein muss. Für den hier betrachteten bilingualen Unterricht beispielsweise hat sich ein fachdidaktischer theoretischer Rahmen erst allmählich entwickelt (Breidbach 2007), sodass das 
unterrichtsbezogene Handeln der Lehrkräfte sich zunächst auf subjektive theoretische Annahmen gründen musste. Damit fehlte für diesen Bereich eine wissenschaftlich abgesicherte theoretische Legitimationsgrundlage. Insgesamt zeigt sich, dass es schwierig ist, ein übergreifendes Professionsmodell auf einen begrenzten Bereich des Lehrerhandelns anzuwenden.

(2) Die „Theorie einer antinomischen Lehrerprofessionalität“ von Helsper (2004: 89) charakterisiert den Lehrberuf als Spannungsfeld einander widersprechender Anforderungen, die jedoch beiderseitig Gültigkeit besitzen. Aus der antinomischen Struktur ergibt sich eine gewisse ,Unmöglichkeit‘ des Lehrberufs, da nie alle Anforderungen gleichzeitig erfüllt werden können. In diesem Bewusstsein verhält sich innerhalb des strukturtheoretischen Ansatzes derjenige professionell, der sich reflexiv mit den Antinomien auseinandersetzt und dem ein situativ stets neu zu leistendes Ausbalancieren der Widersprüchlichkeiten gelingt.

Die Antinomien wirken in fremdsprachendidaktische Entscheidungsfelder hinein und sind für diese spezifiziert worden (Eisenmann/Grimm 2011). Für den bilingualen Unterricht lassen sich zudem widersprüchliche Strukturen beschreiben, die aufgrund der unterschiedlichen beteiligten Fachkulturen zustande kommen. Der bilinguale Unterricht hat sich zwar zu einem eigenständigen Berufsfeld entwickelt, verfügt aber über keine gesicherte grundständige Ausbildungsstruktur. Ein Kriterium, das üblicherweise zugrunde gelegt wird, ist das Vorhandensein von Sachfach- und Fremdsprachenfakultas. Deren ausdrückliche, nicht notwendigerweise aber integrative Verbindung im bilingualen Unterricht kann zu konfligierenden Lehrerbildern, Rollenverständnissen und Handlungsnotwendigkeiten führen, die sich empirisch nachweisen lassen und sich beispielsweise an der Frage des Umgangs mit sprachstrukturellen Aspekten in einem fachlich orientierten Unterricht entzünden (Viebrock 2007, 2010: 114).

(3) Der berufsbiographische Ansatz fokussiert den Prozess der Professionalisierung mit Blick auf Wechselwirkungen mit der privaten Biographie. Er ist narrativ angelegt und berücksichtigt eine longitudinale Perspektive, mithilfe derer er Entwicklungsverläufe von Professionalisierung und De-Professionalisierung rekonstruiert. Bezüglich des bilingualen Unterrichts ist der berufsbiographische Ansatz insofern interessant, als es sich bei dieser Unterrichtsform um eine zunächst in der Schulpraxis initiierte Innovation handelt und sich in der Lehrerschaft bestimmte Typen identifizieren lassen (Dirks 2004), welche dieses Unterrichtskonzept im Kontext bestimmter berufsbiographischer Prozesse entwickeln, tragen und ausgestalten, bzw. auch solche, die sich ihm verweigern.

(4) Von Bedeutung für das hier beschriebene Forschungsprojekt sind darüber hinaus kompetenzorientierte Professionalisierungsmodelle, welche professionelle Kompetenz über das Vorhandensein unterschiedlicher berufsbezogener Voraussetzungen definieren. In dem Modell von Kunter et al. (2011: 59) gehören dazu folgende Kompetenzaspekte: „Professionswissen“, „Überzeugungen“, „motivationale Merkmale“ sowie „,selbstregulative Fähigkeiten“. Das mehrperspektivische Modell von Schratz et al. (2008) hingegen erfasst Professionalität nicht nur als Wissen und Können Einzelner, sondern als Zusammenspiel von personalen und strukturellen Rahmenbedingungen, welche von 
professionellen Lehrenden in selbstreflexiven Prozessen thematisiert werden. Es verbindet individuelle und strukturelle Elemente in fünf „Domänen“: „Reflexivität/Diskursfähigkeit“, „Professionsbewusstsein“, „Kollegialität“, „Differenzfähigkeit“ sowie „Personal Mastery“ (ebd.: 127). Eine ausführliche Diskussion der Leistungsfähigkeit der Modelle kann hier nicht vorgenommen werden. Von Bedeutung ist jedoch, dass das fachdidaktisch relevante Wissen jeweils an unterschiedlicher Stelle verortet wird. Bei Kunter et al. (2011) ist es Teil des Professionswissens. Schratz et al. (2008) entwerfen eine sogenannte ,sechste Disziplin“, welche die fünf Domänen durchzieht und die jeweilige inhaltliche Kontextualisierung (z.B. Fach, Fachdidaktik, Schultyp etc.) umfasst. Während dieser Ansatz aus fachdidaktischer Perspektive sinnhaft erscheint, weil er eine Zuspitzung allgemeiner Professionalisierungsaspekte ermöglicht, bleibt seine Konkretisierung weitgehend diffus.

\section{Subjektive Theorien im Kontext der Professionalisierungsdebatte}

Das Konzept der subjektiven Theorien ist hinsichtlich des theoretischen Rahmens der Professionalisierungsdebatte zunächst offen und lässt sich in den strukturtheoretischen Ansatz ebenso einordnen wie in den kompetenztheoretischen. Auch berufsbiographische Elemente kommen zum Tragen. Unstrittig ist, dass die individuellen Wissensbestände der Lehrkräfte (z.B. in Form subjektiver Theorien) ein wesentliches Element von Professionalität ausmachen.

Ursprünglich waren subjektive Theorien auf kognitive Dispositionen und Wissensbestände fokussiert und wurden in diesem Verständnis als Teil der professionellen Kompetenz von Lehrkräften beschrieben (Lehmann-Grube/Nickolaus 2009), sind dafür aber auch kritisiert worden (Rothland/Brüggemann 2011: 540). Zugleich wird das Konzept in engem Zusammenhang mit ähnlichen Begriffen wie Überzeugungen, teacher beliefs, teachers' mindsets o.ä. diskutiert. Im Gegensatz zu Überlegungen zu einer kollektiven Professionalität im Sinne einer „Berufskultur“ sind subjektive Theorien auf das Individuum bezogen und fragen nicht notwendigerweise nach ,traditionell gewachsenen Formen der Berufsausübung“ (Bennewitz 2008: 248).

Grundsätzlich besteht Einigkeit darüber, dass Lehrerhandeln und professionelle Handlungskompetenz durch subjektive Theorien, individuelle Haltungen oder persönliche Überzeugungen entscheidend beeinflusst werden (Sembill/Seifried 2009: 345, Dann 2000: 88). Die Frage, wie Wissens- und Deutungsbestände der Lehrkräfte produktiv gemacht werden und wie subjektive Theorien die Wissensbasis für beobachtbares professionelles Lehrerhandeln bilden, bleibt allerdings unbeantwortet. Im Gegensatz zu einem kontextualisierten „Herstellungswissen“ (Dann 2000: 89) verfügen subjektive Theorien über einen höheren Abstraktionsgrad. Da sie nicht im Moment der Handlungsbeobachtung erhoben werden können, lässt sich empirisch nicht klären, ob sie tatsächlich handlungsleitend sind oder es sich um nachträgliche Erklärungen und Rechtfertigun- 
gen von Handlungen handelt. Die komplexen Situationskonstellationen pädagogischen Handelns schließen eine direkte Überführung oder deduktive Anwendung regelhafter Wissensbestände ohnehin aus. Vor diesem Hintergrund kommt den subjektiven Theorien eine Orientierungs- und Reflexionsfunktion zu. Von Bedeutung scheint zudem die Organisation der relevanten Wissensbestände (Fachwissen, curriculares Wissen, spezifisches fachdidaktisches Wissen, allgemein pädagogisch-didaktisches Wissen, pädagogisch-psychologisches Wissen) in einer Form zu sein, die eine schnelle Situationserfassung und den Abruf eines entsprechenden Handlungsrepertoires ermöglicht (Rothland/ Brüggemann 2011: 538).

Als weiterer bedeutsamer Einflussfaktor auf die professionelle Handlungskompetenz von Lehrkräften sind das Selbstbild/berufliche Selbstverständnis (Caspari 2003) sowie die berufsbiographische Entwicklung identifiziert worden: „Biographische Reflexionen und überhaupt Reflexivität als Bewusstheit über das eigene Tun wird hier [in den biographisch ausgerichteten Studien] oft als Schlüsselkompetenz von Professionalität aufgefasst" (Combe/Kolbe 2004: 859). Forschungsarbeiten zum bilingualen Unterricht zeigen, dass biographische Untersuchungen (Dirks 2004) im Kern ähnliche Ergebnisse hervorbringen wie Studien zu subjektiven Theorien (Viebrock 2007), auch wenn letztere nicht notwendigerweise explizit nach der biographischen Verankerung der Wissensbestände fragen. Trotzdem lässt sich festhalten: Lehrerhandeln ebenso wie subjektive Theorien im bilingualen Unterricht wurzeln stark in Erfahrungswissen und „,biographisch aufgeschichteten Deutungsbeständen“ (Combe/Kolbe 2004: 859).

Entsprechend lässt sich mit Kunze (2004: 470) das „Lehrersein als lebensgeschichtliche[r] Entwicklungsprozess“" ansehen und das Konzept der subjektiven Theorien im Rahmen des Entwicklungsaufgaben-Ansatzes zur Begleitung der beruflich-professionellen Entwicklung von Lehrkräften und zur Analyse ihrer didaktisch-methodischen Wissensbestände und Handlungskompetenzen produktiv machen. Eine didaktische Entwicklungsaufgabe bezieht sich auf ein didaktisches Entwicklungsziel zwischen einer subjektiven Struktur und ,objektiven“ Anforderungen (Kunze 2004: 473). Die subjektive Struktur umfasst das didaktische Wissen der Lehrkraft, ihre subjektiven didaktischen Theorien als eine spezielle Form des professionellen Wissens sowie ihre didaktische Handlungskompetenz. Die ,objektiven“ Anforderungen beziehen sich auf institutionelle Vorgaben, die Voraussetzungen der Lerner/innen sowie auf ,(gesamt)gesellschaftliche Vorgaben für und Erwartungen an schulischen Unterricht" (ebd.: 473), welche jeweils durch das Subjekt gedeutet und mit der eigenen Verfasstheit abgeglichen werden. Entwicklungsziele werden vom Individuum aufgrund der Feststellung einer Diskrepanz formuliert.

Für den Lehrberuf lassen sich Phasen des Berufslebens unterscheiden, die Kunze zufolge mit beruflichen Entwicklungsaufgaben korrelieren und zudem von, ,allgemeinen lebenslaufbezogenen Entwicklungsaufgaben“ (ebd.: 477), z.B. der Familiengründung, beeinflusst und überlagert werden. Diese Modellbildung ist für das hier beschriebene Forschungsprojekt hilfreich, wenngleich eine genaue Zuordnung bzw. Differenzierung von allgemein beruflichen und spezifisch didaktischen Entwicklungsaufgaben schwierig 
ist. Eine Zuspitzung allgemein beruflicher Entwicklungsaufgaben auf eine (fach-)didaktische Dimension gelingt möglicherweise nur, sieht man Legitimation, unterrichtliche Inszenierung und Reflexion fachlicher und sprachlicher Lernprozesse als Hauptaufgabe der Lehrerarbeit an. Wird das Konzept der subjektiven Theorien an einer Stelle des institutionellen Umbruchs oder der bildungspolitischen Transformation (dazu ließe sich die Einführung des bilingualen Unterrichts zählen, vgl. Eurydice 2006) zur Reflexion eingesetzt, zeigt sich, wie Elemente individueller didaktischer Theorien gefestigt oder hinterfragt werden und wie sich jeweils Stabilisierungen oder Veränderungen in deren Gefüge ergeben (Viebrock 2010).

\section{Bildungspolitische Innovationen und ihr Einfluss auf Professionalisierung}

Das Verhalten von Lehrer/innen in Transformationsprozessen ist insbesondere im Kontext bildungspolitisch motivierter Innovationen mit der Zielvorstellung der Standardisierung untersucht worden (Pant et al. 2008; Maier 2009). Die Ergebnisse sind in Hinblick auf die Professionalisierung der Lehrkräfte, die mit Leung (2009) als prescribed professionalism zu beschreiben wäre, einigermaßen ernüchternd. Maier (2009: 341) kommt in seiner Untersuchung des Einflusses von Vergleichsarbeiten auf das professionelle Selbstverständnis und Handeln zu der Schlussfolgerung, ,dass Lehrkräfte nur zu einem geringen Teil die offiziellen Verlautbarungen zur Funktion der Diagnose- und Vergleichsarbeiten kennen und durchaus eigenwillig den Sinn und Zweck dieser Tests rekonstruieren“. Kritische unterrichtsbezogene Reflexionsprozesse werden kaum angestoßen. Bestimmt man das Maß der Professionalität von Lehrkräften auch in Relation zu den formalen Anforderungen, kommt das Fehlen entsprechender Reflexionsprozesse einer Deprofessionalisierung gleich, da sich der Abstand zwischen erstrebenswerter Handlungsoption und tatsächlicher Handlungsrealisierung vergrößert. Positiv gewendet ließe sich eine eigenwillige Sinnrekonstruktion auch als Ausdruck einer autonomen Auseinandersetzung mit externen Ansprüchen und somit als Ausdruck von Professionalität interpretieren. Allerdings dürfte diese dann nicht uninformiert stattfinden.

Das Ausmaß der Auseinandersetzung mit der Top-down verordneten Implementierung länderübergreifender Bildungsstandards untersuchen Pant et al. (2008). Als wesentlichen Erfolgsfaktor für das Gelingen eines Reformprozesses setzen sie ,einen Mindestgrad an Akzeptanz der Konzepte und Maßnahmen durch die Lehrerinnen und Lehrer als die zentralen Akteure“ (ebd.: 827) voraus. Dieser ist nicht unbedingt gegeben, da mit der Hinwendung zu kompetenzorientierten, standardbasierten Unterrichtsformen ein deutlicher Evaluationsdruck entsteht, welcher wiederum Auswirkungen auf das professionelle Selbstverständnis hat. Relativ kurz nach der Einführung der Bildungsstandards lassen sich vor allem selbstbezogene Überlegungen zur Auswirkung der Reform auf die eigene Person/Rolle nachweisen. Nicht belegen lassen sich unterrichtsbezogene Überlegungen im engeren Sinne (z.B. hinsichtlich der Materialbeschaffenheit oder Me- 
thodenwahl): „Die vergleichsweise geringe Beschäftigung mit den alltagspraktischen Aspekten standardbasierten Unterrichtens mag als Hinweis darauf gelesen werden, dass viele Lehrkräfte in Deutschland derzeit solche Unterrichtsformen entweder noch gar nicht identifizieren können oder sie zurzeit als eine zunächst nur theoretische Innovationsanforderung begreifen“ (ebd.: 841).

Bennewitz (2008) kommt in ihrer Studie des Einflusses der Einführung der reformpädagogisch orientierten Förderstufe in Sachsen-Anhalt auf die Deutungsmuster der Lehrenden zu vergleichbaren Ergebnissen, was die Übernahme von Verantwortung für Innovationsprozesse betrifft: ,Schulreform wirkt nur dann bestärkend - als Handlungsaufforderung hin zu neuen Formen des Unterrichtens - wenn sie mit individuellen Veränderungsprozessen in zeitlicher und in inhaltlicher Übereinstimmung zusammentrifft" (ebd.: 257). Anstelle von individuellen Veränderungsprozessen ließe sich mit Kunze (2004) auch von beruflichen/didaktischen Entwicklungsstadien und den darin jeweils zu bearbeitenden Aufgaben sprechen. Die kurze Zusammenschau der Studien top-downinitiierter Schul-/Unterrichtsentwicklungsprozesse zeigt, dass sie trotz höchst unterschiedlicher Designs und methodologischer Grundannahmen übereinstimmende Ergebnisse hervorbringen, welche berechtigte Zweifel an der positiven Wirkung aufgesetzter bildungspolitischer Innovationen auf Professionalisierungsprozesse von Lehrkräften aufkommen lassen. Das Spannungsfeld von administrativer Kontrolle und professioneller Autonomie scheint zumindest in den frühen Stadien der Reformen teilweise mithilfe von Nichtbeachtung aufgelöst zu werden.

Die Einführung des bilingualen Unterrichts ist zwar auch von äußerst optimistischer, nicht notwendigerweise empirisch belegter bildungspolitischer Rhetorik begleitet (vgl. dazu Bonnet 2012); sie kann im Gegensatz zu den zuvor diskutierten Beispielen aber als Bottom-Up-Prozess gelten, der aus der Initiative engagierter Lehrer/innen hervorgegangen und somit Charakteristikum eines independent professionalism (Leung 2009) ist. Die Entscheidung für ein neues Bildungsangebot erzeugt zwar auch Handlungsdruck, dieser wird im Gegensatz zu den verordneten Reformen allerdings von den Lehrkräften selbst verantwortet. Die innovationeinführenden Instanzen können nicht oder nur im Einzelfall, wenn die Initiative zum Beispiel ausschließlich von der Schulleitung ausgeht, als Gegenspieler der Lehrerschaft konzipiert werden. Daher kann vermutet werden, dass sich die Herausforderungen eher als sinnstiftend, weniger als kontrollierend, einengend oder anstrengend erweisen. Die Entwicklung bilingualer Unterrichtsangebote und der damit verbundene Gestaltungsspielraum lassen sich aus Sicht der beteiligten Lehrkräfte möglicherweise insgesamt als Ausdruck von Autonomie interpretieren.

Mit Blick auf die genannten Beispiele ist nicht nur die Dimension prescribed independent zu unterscheiden, sondern auch die Reichweite der jeweiligen Innovation. Nach Altrichter/Wiesinger (2005: 32) streben Innovationen Veränderungen auf vier Ebenen an: „Praktiken“, „unterliegendes Wissen und Einstellungen“, deren „,materielle Aspekte“ sowie die umgebenden ,,sozialen und organisationalen Strukturen“. Im Implementierungsprozess ergeben sich zwei Ansätze: der ,programmierte“ und der ,adaptivevolutionäre“ (ebd.), die jeweils vom Ausmaß der Veränderung, der Einstellung der Ak- 
teure, der Stabilität des Umfelds und weiteren Indikatoren abhängen. Es ließe sich vor dem Hintergrund dieses Modells argumentieren, dass die Entwicklung des bilingualen Unterrichts eher den Charakteristika des programmierten Ansatzes zuzuordnen ist, da übergeordnete institutionelle Organisationsstrukturen und vor allem soziale (Macht-) Strukturen kaum berührt werden, die angestrebte Innovation von den beteiligten Akteuren positiv gesehen wird und zudem innerhalb eines grundsätzlich bekannten Formats (z.B. auf der Ebene der Unterrichtsmethoden) stattfindet. Die anderen Reformen hingegen sind aufgrund der Tatsache, dass sie die gesamte Lehrerschaft sowie in erster Linie soziale und organisationale Strukturen ansprechen und erst nachgeordnet Einfluss auf die weiteren Ebenen von Innovation ausüben, von deutlich größerer Reichweite. Sie sind demnach dem adaptiv-evolutionären Ansatz zuzuordnen, der die Implementierung von Innovationen als Entwicklungsprozess betrachtet, der von den Akteuren auf allen Ebenen beeinflusst wird und notwendigerweise zu Modifikationen der ursprünglichen Innovation führt.

\section{Subjektive didaktische Theorien von bilingualen Erdkundelehrer/innen}

Nachdem dargelegt wurde, wie sich das Konzept der subjektiven Theorien zur Professionalisierungsdebatte verhält und in welchen Punkten sich der bilinguale Unterricht von verordneten Innovationen unterscheidet bzw. diesen ähnelt, sollen ausgewählte Ergebnisse des Forschungsprojekts erläutert werden.

Die subjektiven didaktischen Theorien von zehn bilingualen Erdkundelehrer/innen wurden mithilfe von problemzentrierten, leitfadengestützten Interviews (Witzel 1996) erhoben sowie kommunikativ (feedback paper) und argumentativ (Unterrichtsbeobachtung, erneutes Interview) validiert. Die Forschungsteilnehmer/innen wurden nach den Leitlinien des theoretical sampling (Strauss/Corbin 1996) hauptsächlich mithilfe von gatekeepers (Merkens 2003: 288) akquiriert. Zur systematischen Auswertung der transkribierten Daten dienten Sequenz-, Argumentations- und Metaphernanalysen (Viebrock 2007: 101ff.). Während die Sequenzanalyse zunächst dazu diente, in chronologischer Reihenfolge die thematisierten Konzepte herauszuarbeiten und erste inhaltliche Interpretationen vorzunehmen, diente die Argumentationsanalyse zur Herausarbeitung von Makrostrukturen des Argumentationsprozesses. In beiden Fällen wurden umfangreiche Fragenkataloge an das Material herangetragen und dieses damit systematisch analysiert. Ergänzend wurde eine Metaphernanalyse durchgeführt, um einen Zugang zu mentalen Konzepten, Strukturen und Modellvorstellungen zu bekommen. Strukturskizzierungen wurden in Abgrenzung zu Groeben et al. (1988) im Prozess der Analyse ausschließlich von der Forscherin ohne formelles Regelwerk eingesetzt, um Zusammenhänge zwischen einzelnen Konzepten (z.B. Wenn-dann-Relationen) sichtbar zu machen (Viebrock 2004). Für die kommunikative Validierung sowie die Ergebnisdarstellung wurden die Skizzierungen wieder in einen Text überführt. 
Wenngleich es schwierig ist, mit wenigen Datenbeispielen der Komplexität der subjektiven Theoriegebilde gerecht zu werden, soll mithilfe der folgenden Transkriptauszüge aus einer Fallstudie illustriert werden, wie subjektive Theorieelemente aus dem Datenmaterial herausgearbeitet wurden. Die Aussagen stammen aus dem Interviewbeginn. Die angesprochenen Thematiken werden auch an anderen Stellen des Interviews weiter ausgeführt.

(I) /äh/- ich hatte, muss ich dazu sagen, zunächst einmal doch recht negative Erfahrungen gemacht mit dem Schulbuch, mit dem englischen Schulbuch. (ja, das ist dieses /äh/ von [Name des Verlags], oder?) Das war [Name des Schulbuchs], ja, das sehr stark textorientiert ist (ja) ja, und /äh/ vor allen Dingen /äh/ sah ich den Nachteil dieses Schulbuchs eben darin, dass die Texte sehr konstruiert waren (ja) das war häufig so, dass da ein längerer Text war auch mit landeskundlichem Inhalt oder auch /äh/, ja, mit anderen Inhalten, aber dann wurde beispielsweise- dieser Text wurde verwendet, /äh/ um irgendwelche grammatischen Phänomene zu transportieren und so ( $\mathrm{hmhm}, \mathrm{hmhm}$ ) so entwickelte also dieser Unterricht teilweise recht recht zäh und recht langweilig (hmhm) und war auch für die Schüler wenig motivierend, und /äh/ jetzt sah ich im bilingualen Unterricht da Möglichkeiten, auch /äh/, ja, anders zu unterrichten einmal (hmhm) viel mehr eben, auch gerade was den Anfangsunterricht anbelangt $(\mathrm{hmhm})$, /äh/ anschaulich zu arbeiten $(\mathrm{hmhm})$ die Notwendigkeit, anschaulich zu arbeiten.

(II) Wobei, und da kommen wir später vielleicht auch noch drauf, man reduzieren muss (ja) im bilingualen Unterricht, das ist ganz klar. Es kommt auch meinen Neigungen entgegen, meinen didaktischen Neigungen sozusagen, exemplarisch zu unterrichten, (ja) sich auf das Wesentliche zu konzentrieren, $(\mathrm{hmhm})$ und /äh/ dass ich eben auch einen groß- eine große Möglichkeit hier in- in diesem Fach.

(III) Und gerade auch dieses methodisches Aus- das das fand ich sehr wichtig (hmhm) und insofern sind das /äh/- sicherlich auch damals, als wir noch mit dem alten Buch arbeiteten, hab ich also verstärkt dann auch Wert gelegt auf, ja auf diese methodischen Dinge, $(\mathrm{hmhm})$ nech, dass man den Schülern auch /äh/ da das Arbeits- /äh/ Vokabular vermittelt, das notwendig ist, nicht nur um Texte auszuwerten, was ja sonst immer der Fall ist, sondern beispielsweise auch um Bilder, Grafiken, Statistiken (hmhm, hmhm) und so weiter auszuwerten.

Die Analyse der ersten Äußerung, welche als Antwort auf die Frage nach den Gründen des persönlichen Engagements für den bilingualen Unterricht gegeben wurde, zeigt, dass der bilinguale Unterricht ex negativo in Abgrenzung zum grundständigen, durch die Transportmetapher als hochgradig formorientiert charakterisierten Fremdsprachenunterricht definiert wird. Trotz potenziell ansprechender Textinhalte bleibe der eigentliche Unterrichtszweck ein focus on form. Diese starke Text-/Formorientierung im Fremdsprachenunterricht wird explizit in einen Kausalzusammenhang mit einer ungünstigen Motivationslage sowohl auf Seiten der Lehrkraft (,zähe und langweilige Unterrichtsentwicklung“) als auch auf Seiten der Schüler/innen (,wenig motivierend“) gestellt. Implizit wird damit das Motivationspotenzial des bilingualen Unterrichts positiv markiert und kurze Zeit später im Interview durch eine Charakterisierung der Materialen als ,,authentischer" bestärkt.

Im dritten Auszug rücken fachmethodische und sprachliche Aspekte sowie deren Verbindung in den Mittelpunkt. Dem bilingualen Erdkundeunterricht wird eine größere Vielfalt der Textsorten (v.a. diskontinuierliche Texte: „Bilder, Graphiken, Statistiken“) zugeschrieben. Diese ist wiederum positiv konnotiert, weil sie abweicht vom grundstän- 
digen, aus Sicht des Befragten als stark textbasiert konzeptionalisierten Fremdsprachenunterricht (,wie sonst immer der Fall“). Mit Blick auf die persönliche Entwicklung der Lehrperson kommt dem bilingualen Unterricht somit eine Katalysatorfunktion zu, ,einmal anders zu unterrichten“ und Alternativen zu einer starken Textorientierung zu finden. Das Prinzip der „Anschaulichkeit“, das sich mit Blick auf das gesamte Interviewtranskript als zentrales didaktisches Prinzip bestimmen lässt, wird zum ersten Mal benannt.

Im zweiten Interviewauszug werden weitere Grundsätze angeführt: eine Reduktion bzw. Konzentration auf ,das Wesentliche“ sowie eine Orientierung am Prinzip des Exemplarischen. Auffällig ist, dass diese in ähnlicher Weise als Imperativ an die Lehrkraft formuliert werden (,,man muss“") wie im ersten Abschnitt die Wahrnehmung, sich der im Englischunterricht vorgegebenen Formorientierung des Lehrbuchs nicht entziehen zu können. Während der bilinguale Unterricht also eine Alternative zu den vermeintlichen Imperativen des Englischunterrichts darstellt, unterliegt er zugleich seinen eigenen. In der vorliegenden Fallstudie sind diese allerdings nicht negativ konnotiert, da sie den „didaktischen Neigungen“ des Befragten entsprechen.

In der Zusammenschau ergeben sich erste Leitlinien einer subjektiven didaktischen Theorie des Befragten zum bilingualen Erdkundeunterricht, die auch im Fachunterricht geltende Prinzipien in den Mittelpunkt rückt: z.B. Anschaulichkeit und das Prinzip des Exemplarischen. Das Spannungsverhältnis zwischen kognitiver Leistungsfähigkeit der Lernenden und ihren Möglichkeiten, sich fremdsprachlich auszudrücken, schafft ein Bewusstsein für die Notwendigkeit eines sprachlichen Scaffolding. Dieses ist eng verknüpft mit einem Methodenbewusstsein: fachspezifische Arbeitsmethoden umfassen zugleich die Fähigkeit, diese angemessen zu verbalisieren. Insgesamt wird das vermeintliche Potenzial des bilingualen Unterrichts - insbesondere durch seine Abgrenzung zum grundständigen Fremdsprachenunterricht - positiv überhöht.

Das gewählte Beispiel illustriert, wie Lehrkräfte durch die innovative Unterrichtsform des bilingualen Unterrichts insbesondere in ihrer methodischen Planung, zum Teil auch hinsichtlich didaktischer Überlegungen zu einem ,Re-Framing etablierter Praktiken“" (Dirks 2004: 136) angeregt werden. Die geteilten und routinemäßig verfügbaren Wissensbestände einer monolingual deutschsprachigen Fach-/Unterrichtstradition werden zugunsten einer bewussteren methodischen Inszenierung und teilweise auch veränderten Systematisierung von Unterrichtsgegenständen aufgebrochen, die durch die Notwendigkeit ausgelöst wird, fachliche Erkenntnisprozesse im Medium der Fremdsprache zu inszenieren. Inspiration bieten auch internationale Fach- und Unterrichtskulturen, z.B. bei der Übernahme von Methodiken aus dem angelsächsischen Raum. Das überwiegend methodische Re-Framing geht auch in umfassenderes Reflecting über, das über das Fach hinaus rückwirkt und dem man Potenzial für die Ausbildung einer „didaktischen Grundlagenbewusstheit“ hinsichtlich der sprachlichen Verfasstheit aller Lernprozesse zuschreiben kann. Allerdings stellt sich diese nicht notwendigerweise ohne Anleitung ein.

Eine Zusammenschau der Inhalte und Strukturen der erhobenen subjektiven Theorien zeigt zudem, dass der bilinguale Unterricht mit einzelnen Ausnahmen als Sach- 
fachunterricht in der Fremdsprache konzeptionalisiert wird. Seine zentrale Aufgabe wird darin gesehen, feststehende Inhalte des Sachfachs fremdsprachlich zu ,,vermitteln“. Dieses Verständnis bilingualen Unterrichts bezeichnet Breidbach (2007: 66ff.) als „Vehikularsprachenmodell“. Dessen Grundlage ist die Annahme einer Unabhängigkeit der Lernprozesse von einer spezifischen Sprache. Es beruft sich auf eine inhaltliche und kommunikative Authentizität des Unterrichts, die sich in der Logik des Modells unweigerlich aus einer mitteilungsorientierten Kommunikation ergibt, ohne dass eine explizite Legitimation der sachfachtypischen Inhalte vorgenommen werden muss. Der Erwerb fremdsprachlicher Kompetenzen wird in diesem Verständnis als willkommener „Mehrwert“ angesehen, der sich gleichsam automatisch als Nebeneffekt ergibt. Davon abgesehen, dass die Mehrwert-Metaphorik einer empirischen Grundlage entbehrt und inzwischen äußerst kritisch diskutiert wird (Bonnet 2012), stellt sich in diesem Zusammenhang die Frage, in welchem Maß die Konzentration auf sachfachliche Inhalte mit einer Authentizität der Kommunikation einhergeht. Hier scheint die Annahme vorzuliegen, dass Kommunikation immer als natürlich und authentisch zu gelten hat, sobald die Fremdsprache und ihre Strukturen nicht explizit thematisiert werden. Zwar mögen die Fachinhalte authentisch für den Unterricht sein, über ihre lebensweltliche Bedeutung für die Lerner ist damit aber noch nichts gesagt. Ein weiteres typisches alltagstheoretisches Argumentationsmuster für den bilingualen Unterricht in diesem Zusammenhang ist die stärkere kognitive Verankerung von Fachbegriffen im Sinne einer höheren „Verarbeitungstiefe" durch den Gebrauch von zwei Sprachen. Der empirische Nachweis dieser Annahme steht noch aus.

Mit Blick auf die angenommene positive Wirkung von Bottom- $U p$-Innovationen auf die professionelle Handlungskompetenz der Lehrkräfte ist ein weiterer Aspekt der subjektiven Theorien bedeutsam: Die Wahrnehmung erweiterter sprachlicher Anforderungen im bilingualen Unterricht wird zur Rechtfertigung von Lehrerzentrierung (insbesondere in der Funktion als fremdsprachliches role model) herangezogen. Es ist zu hinterfragen, ob der bilinguale Unterricht Gefahr läuft, zu überholten instruktivistischen Unterrichtsformen und lehrerzentrierten Rollenbildern zurückzukehren, und damit Bedenken bestätigt, die Decke-Cornill (1999: 167) bereits in der frühen Expansionsphase formuliert hat.

\section{Fazit}

Zusammenfassend zeigen die Ergebnisse des Forschungsprojekts, dass eine bottom-upinitiierte Innovation wie der bilinguale Unterricht zwar ein Potenzial zur Reflexion und Aufbrechung didaktischer und methodischer Routinen beinhaltet. Somit leistet diese Unterrichtsform einen wichtigen Beitrag zur individuellen didaktisch-methodischen Professionalisierung der Lehrkräfte. Zugleich werden Reflexionspotenziale aber durch persönlichkeitsbezogene Eigenschaften (z.B. den grundsätzlichen Unterrichtsstil) sowie pragmatische Anforderungen (z.B. die Erstellung von sprachlich sowie fachlich ange- 
messenem Unterrichtsmaterial) überlagert. Die forschungsmethodische Umsetzung des Konzepts der subjektiven Theorien in der vorliegenden Studie (d.h. Interview, feedback paper, Unterrichtsbeobachtung, erneutes Interview) stellt auch aus Sicht der Befragten eine geeignete Möglichkeit dar, die Potenziale ebenso wie Grenzen sichtbar zu machen und Weiterentwicklungen auch in der von Schratz et al. (2008) geforderten institutionalisierten Form anzustoßen (Viebrock 2007: 71).

\section{Autorenangaben}

Prof. Dr. Britta Viebrock

Fachbereich 10, Institut für England- und Amerikastudien

Goethe-Universität Frankfurt/Main

viebrock@em.uni-frankfurt.de

\section{Literatur}

Altrichter, H./Wiesinger, S. (2005): Implementation von Schulinnovationen - aktuelle Hoffnungen und Forschungswissen. In: Journal für Schulentwicklung 9, 4, S. 28-36.

Bennewitz, H. (2008): Lehrende in Schulreformprozessen: Eine Deutungsmusteranalyse. In: Breidenstein, G./Schütze, F. (Hrsg.): Paradoxien in der Reform der Schule. Ergebnisse qualitativer Forschung. Wiesbaden: VS Verlag für Sozialwissenschaften, S. 247-260.

Bonnet, A. (2012): Towards an evidence base for CLIL: How to integrate qualitative and quantitative as well as process and product perspectives in CLIL research. In: International CLIL Research Journal, 1, S. 66-78. Online: www.icrj.eu (letzter Zugriff 20.12.2013).

Breidbach, S. (2007): Bildung, Kultur, Wissenschaft. Reflexive Didaktik für den bilingualen Sachfachunterricht. Münster: Waxmann.

Breidbach, S./Viebrock, B. (2012): CLIL in Germany - Results from recent research in a contested field of education. In: International CLIL Research Journal, 1, S. 5-16. Online: www.icrj.eu (letzter Zugriff 20.12.2013).

Caspari, D. (2003): Fremdsprachenlehrerinnen und Fremdsprachenlehrer. Studien zu ihrem beruflichen Selbstverständnis. Tübingen: Narr.

Combe, A./Kolbe, F.-U. (2004): Lehrerprofessionalität: Wissen, Können, Handeln. In: Helsper, W./Böhme, J. (Hrsg.): Handbuch der Schulforschung. Wiesbaden: VS Verlag für Sozialwissenschaften, S. 833-851.

Dann, H.-D. (2000): Lehrerkognitionen und Handlungsentscheidungen. In: Schweers, M./Martin, K. W. (Hrsg.): Lehrer-Schüler-Interaktion. Pädagogisch-psychologische Aspekte des Lehrens und Lernens in der Schule. Opladen: Leske + Budrich, S. 79-108.

Decke-Cornill, H. (1999): Einige Bedenken angesichts eines möglichen Aufbruchs des Fremdsprachenunterrichts in eine bilinguale Zukunft. In: Neusprachliche Mitteilungen, 3, S. 164-170.

Dirks, U. (2000): Wie werden EnglischlehrerInnen professionell? Eine berufsbiographische Untersuchung in den neuen Bundesländern. Münster: Waxmann.

Dirks, U. (2004): Kulturhüter oder Weltenwanderer? Zwei „ideale“ Realtypen bilingualen Sachfachunterrichts. In: Bonnet, A./Breidbach, S. (Hrsg.): Didaktiken im Dialog. Konzepte des Lehrens und Wege des Lernens im bilingualen Sachfachunterricht. Frankfurt/M.: Lang, S. 129-140. 
Eisenmann, M./Grimm, T. (Hrsg.) (2011): Heterogene Klassen - Differenzierung in Schule und Unterricht. Baltmannsweiler: Schneider Hohengehren.

Eurydice (Hrsg.) (2006): Content and Language Integrated Learning (CLIL) at School in Europe. Brüssel: Eurydice: The Information Network on Education in Europe.

Groeben, N./Wahl, D./Schlee, J./Scheele, B. (1988): Forschungsprogramm Subjektive Theorien. Eine Einführung in die Psychologie des reflexiven Subjekts. Tübingen: Francke.

Helsper, W. (2004): Antinomien, Widersprüche, Paradoxien: Lehrerarbeit - ein unmögliches Geschäft? Eine strukturtheoretisch-rekonstruktive Perspektive auf das Lehrerhandeln. In: Koch-Priewe, B./Kolbe, F.-U./Wildt, J. (Hrsg.): Grundlagenforschung und mikrodidaktische Reformansätze zur Lehrerbildung. Bad Heilbrunn: Klinkhardt, S. 49-99.

Kunter, M./Kleickmann, T./Klusmann, U./Richter, D. (2011): Die Entwicklung professioneller Kompetenz von Lehrkräften. In: Kunter, M./Baumert, J./Blum, W./Klusmann, U./Krauss, S./Neubrand, M. (Hrsg.): Professionelle Kompetenz von Lehrkräften. Ergebnisse des Forschungsprogramms COACTIV. Münster: Waxmann, S. 55-68.

Kunze, I. (2004): Konzepte von Deutschunterricht. Eine Studie zu individuellen didaktischen Theorien von Lehrerinnen und Lehrern. Wiesbaden: VS Verlag für Sozialwissenschaften.

Lehmann-Grube, S. K./Nickolaus, R. (2009): Professionalität als kognitive Disposition. In: Zlatkin-Troitschanskaia, O./Beck, K./Sembill, D./Nickolaus, R./Mulder, R. (Hrsg.): Lehrprofessionalität. Bedingungen, Genese, Wirkungen und ihre Messung. Weinheim, Basel: Beltz, S. 59-70.

Leung, C. (2009): Second language teacher professionalism. In: Burns, A./Richards, J. C. (Hrsg.): The Cambridge guide to second language teacher education. Cambridge: Cambridge University Press. S. 49-58.

Maier, U. (2009): Wie gehen Lehrerinnen und Lehrer mit Vergleichsarbeiten um? Eine Studie zur testbasierten Schulreform in Baden-Württemberg und Thüringen. Baltmannsweiler: Schneider Verlag Hohengehren.

Merkens, H. (2003): Auswahlverfahren, Sampling, Fallkonstruktion. In: Flick, U./Kardorff, E. von/Steinke, I. (Hrsg.): Qualitative Forschung. Ein Handbuch. 2. Auflage. Reinbek bei Hamburg: Rowohlt, S. 286-299.

Pant, H. A./Vock, M./Pöhlmann, C./Köller, O. (2008): Offenheit für Innovationen. Befunde aus einer Studie zur Rezeption von Bildungsstandards bei Lehrkräften und Zusammenhänge mit Schülerleistungen. In: Zeitschrift für Pädagogik 54/6, S. 827-845.

Rothland, M./Brüggemann, T. (2011): Professionalität und Lehrerkompetenz. In: Sandfuchs, U./ Melzer, W./Dühlmeier, B./Rausch, A. (Hrsg.): Handbuch Erziehung. Bad Heilbrunn: Klinkhardt UTB, S. 536-541.

Schratz, M./Schrittesser, I./Forthuber, P./Pahr, G./Paseka, A./Seel, A. (2008): Domänen der Lehrer/innen/professionalität: Rahmen einer kompetenzorientierten Lehrer/innen/bildung. In: Kraler, C./Schratz, M. (Hrsg.): Wissen erwerben, Kompetenzen entwickeln. Münster: Waxmann, S. 123-138.

Sembill, D./Seifried, J. (2009): Konzeptionen, Funktionen und intentionale Veränderungen von Sichtweisen. In: Zlatkin-Troitschanskaia, O./Beck, K./Sembill, D./Nickolaus, R./Mulder, R. (Hrsg.): Lehrprofessionalität. Bedingungen, Genese, Wirkungen und ihre Messung. Weinheim/Basel: Beltz, S. 345-354.

Strauss, A./Corbin, J. (1996): Grounded Theory. Grundlagen Qualitativer Sozialforschung. Weinheim: Beltz, Psychologie Verlags Union.

Viebrock, B. (2004): Elemente einer subjektiven didaktischen Theorie: Was ein Lehrer über fremdsprachliches Lernen und Konzeptbildung im bilingualen Erdkundeunterricht denkt. In: Bonnet, A./Breidbach, S. (Hrsg.): Didaktiken im Dialog. Frankfurt/M.: Lang, S. 167-178.

Viebrock, B. (2007): Bilingualer Erdkundeunterricht. Subjektive didaktische Theorien von Lehrerinnen und Lehrern. Frankfurt/M.: Lang. 
Viebrock, B. (2010): Alltagstheorien, methodisches Wissen und unterrichtliches Handeln. In: Doff, S. (Hrsg.): Bilingualer Sachfachunterricht in der Sekundarstufe. Eine Einführung. Tübingen: Narr, S. 107-123.

Witzel, A. (1996): Auswertung problemzentrierter Interviews. Grundlagen und Erfahrungen. In: Strobl, R./Böttger, A. (Hrsg.) (1996): Wahre Geschichten? Zur Theorie und Praxis qualitativer Interviews. Baden-Baden: Nomos, S. 49-75. 